\title{
Editorial
}

\section{Diagnostic Laparoscopy in Infertility}

\author{
Samsad Jahan \\ Professor \\ Department of Obstetrics \& Gynaecology \\ BIRDEM, Dhaka, Bangladesh
}

Laparoscopy is a minimally invasive surgical technique that provides a panoramic and magnified view of the pelvic organs and peritoneal surfaces and allows surgery at the time of diagnosis. ${ }^{1}$ Historically, only diagnostic procedures were performed with the laparoscope. In the last decade, use of the laparoscope has expanded. Now various reproductive disorders are diagnosed and treated primarily with the laparoscope including pelvic adhesions, endometriosis, and disorders of the fallopian tubes. In response to the significant advances in endoscopy, today's gynecologic surgeons can integrate operative laparoscopy into daily practice and in many situations. Infertility is a major health issue with multifactorial aetiology. None of the laboratory findings alone is sufficient in diagnosing infertility. Laparoscopy provides important and essential information helpful in the investigation and management of infertility. ${ }^{2}$

Laparoscopy is generally regarded as the most reliable tool in the diagnosis of tubal pathology and other intra-abdominal causes of infertility. ${ }^{1}$ Where there is no suggestive clinical signs and symptoms, laparoscopy offers an excellent means through direct visualization. Diagnostic laparoscopy (DLS) is generally accepted as the most accurate procedure to detect tubal pathology and endometriosis but it is still a matter of debate whether laparoscopy should be routinely done in the infertility work-up. ${ }^{3}$ There are several non invasive and cost effective tools to evaluate or predict tubal pathology. Several studies describe the accuracy of Chlamydia antibody testing (CAT) and hysterosalpingography (HSG) with diagnostic laparoscopy as gold standard but alone no one tool is conclusive. ${ }^{4}$
Hysterosalpingography has been classically used for decades for the evaluation of tuboperitoneal infertility. Other newer ultrasonography-based modalities like hysterosalpingo contrast sonography (HyCoSy) and saline intraperitoneal sonogram (SIPS) have evolved which are safe, quick, and cost effective and also avoid exposure to X-rays. Based on symptoms suggestive of previous pelvic inflammatory disease (PID), a history of abnormal vaginal discharge and a previous diagnosis of a lower genital tract infection, the positive predictive value of thorough history taking, was only $56 \%, 59 \%$, and $35 \%$, respectively, in predicting tuboperitoneal infertility in several studies. ${ }^{1,5,6} \mathrm{~A}$ meta-analysis of 20 studies comparing HSG and laparoscopy for tubal patency and peritubal adhesions showed that HSG has low sensitivity for tubal patency, but high specificity for confirming the presence of tubal obstruction. For the evaluation of peritubal adhesions and especially endometriosis, HSG is not reliable and requires laparoscopy. ${ }^{7}$ Laparoscopy still reveals tubal pathology or endometriosis in 35-68\% of cases, even after normal HSG and in patients with no suggestive history. 7,8

Many women with pelvic endometriosis however are asymptomatic. Lack of satisfactory non-invasive tests for endometriosis has made laparoscopy the gold standard for diagnosis. ${ }^{1}$ Laparoscopy also has therapeutic role in endometriosis. In a recent Cochrane review, Jacobson and co-workers concluded that use of laparoscopic surgery in the treatment of subfertility related to minimal and mild endometriosis may improve future fertility. ${ }^{9}$ However, no RCTs or meta-analyses are available for laparoscopic treatment of moderate and severe 
endometriosis, albeit it is generally accepted that those should be treated by surgery. ${ }^{10}$ A negative correlation between the stage of endometriosis and the spontaneous cumulative pregnancy rate (CPR) after surgical management has been reported. ${ }^{11}$ Regarding laparoscopic adhesiolysis, only one non-randomized controlled study documented higher CPR after the procedure. ${ }^{12}$

As Chlamydia trachomatis is the most important aetiologic factor in PID, screening for Chlamydia antibodies (by Chlamydia antibody testing or CAT) has been proposed as a primary screening tool for infertility due to tubal pathology. ${ }^{13} \mathrm{~A}$ meta-analysis of studies comparing Chlamydia antibody titres and laparoscopy for tubal patency and peritubal adhesions has shown that the discriminative capacity of Chlamydia antibody titres in the diagnosis of any tubal pathology is comparable to that of HSG in the diagnosis of tubal occlusion. ${ }^{4}$ Although CAT can be determined at low cost, it fails to provide information about the severity of tubal pathology, which is of importance to fertility prognosis and, subsequently, to infertility treatment. Furthermore, it cannot detect tubal pathology due to other causes or endometriosis.

According to World Health Organization (WHO) guidelines, DLS is still recommended as a minimal requirement in the investigation of infertility in the female. ${ }^{14}$ However, it remains questionable whether DLS in general provides more information to further diagnosis and treatment decisions. There is a growing tendency to bypass diagnostic laparoscopy in couples with anormal HSG who will undergo intrauterine insemination (IUI) treatment for idiopathic infertility, mild male subfertility and cervical hostility.

Generally, laparoscopy should be reserved for couples who have already completed a more basic infertility evaluation including assessing for ovulation, ovarian reserve, ultrasound and hysterosalpingogram for the female and semen analysis for the male. Some couples may choose to skip laparoscopy and proceed to other fertility treatments such as superovulation with fertility medications combined with intrauterine insemination or in vitro fertilization.

The purpose of the diagnostic laparoscopy is first to trace abnormalities and secondly to treat them when necessary. Diagnostic laparoscopy reveals that tubal lesions and pelvic adhesions are still the major causes for female infertility in developing countries. Operative procedures, such as adhesiolysis, ablation of endometriosis, tuboplasty and salpingectomy for hydrosalpinx or pyosalpinx at the time of laparoscopy can enhance conception, naturally or with intra uterine insemination or in vitro fertilization. ${ }^{1}$ Therefore, training in endoscopic surgery should be regarded as an important issue in developing countries. Besides the training in endoscopy surgery, it is urgent to develop a preventive strategy of genital tract infection to reduce the risk of tubo-peritoneal lesions.

Delta Med Col J. Jan 2016;4(1):1-3

\section{References}

1. Ngowa JDK, Kasia JM, Nkongo V, NgassamA, Tsuala JF, Nsem P, et al. Contribution of Laparoscopy in the Management of Female Infertility in Low Resource Countries: A Review of 208 Cases at the Yaoundé General Hospital, Cameroon. IJCM. 2015;6(12):934-39.

2. Patton P. Operative Laparoscopy for Infertility. Glob Libr Women's Med. 2008; DOI 10.3843/GLOWM.10332.

3. Tanahatoe SJ, Hompes PGA, Lambalk CB. Accuracy of Diagnostic Laparoscopy in the Infertility Work-up before Intrauterine Insemination. Fertil Steril. 2003b;79:361-66.

4. Mol BW, Dijkman B, Wertheim P, Lijmer J, van der Veen F, Bossuyt PM. The Accuracy of Serum Chlamydial Antibodies in the Diagnosis of Tubal Pathology: A Meta Analysis. Fertil Steril. 1997;67:1031-37.

5. Berker B, Mahdavi A, Shahmohamady B, Nezhat C. Role of Laparoscopic Surgery in Infertility. Middle East Fertility Society Journal. 2005;10:94-104. 
6. Fouany MR, Muasher SJ. Is There a Role for Laparoscopy in the Diagnosis and Treatment of Infertility in the $21^{\text {st }}$ Century? Middle East Fertility Society Journal. 2010;15:146-52.

7. Swart P, Mol BW, van der Veen F, van Beurden M, Redekop WK, Bossuyt PM. The Accuracy of Hysterosalpingography in the Diagnosis of Tubal Pathology: A Meta-Analysis. Fertil Steril. 1995;64:486-91.

8. Corson SL, Cheng A, Gutmann JN. Laparoscopy in the 'Normal' Infertile Patient: A Question Revisited. J Am Assoc Gynecol Laparosc. 2000;7:317-24.

9. Jacobson TZ, Duffy JM, Barlow D, Farquhar C, Koninckx PR, Olive D. Laparoscopic Surgery for Subfertility Associated with Endometriosis. Cochrane Database Syst Rev. 2010;1:CD001398.

10. Kennedy S, Bergqvist A, Chapron C, D'Hooghe T, Dunselman G, Greb R, et al. ESHRE Guidelines for the Diagnosis and Treatment of Endometriosis. Hum Reprod. 2005;20:2698-704.
11. Bosteels J, van Herendael B, Weyers S, D’Hooghe T. The Position of Diagnostic Laparoscopy in Current Fertility Practice. Hum Reprod Update. 2007; 13:477-85.

12. Tulandi T, Collins JA, Burrows E, Jarrell JF, McInnes RA, Wrixon W. Treatment-Dependent and Treatment-Independent Pregnancy among Women with Periadnexal Adhesions. Am J Obstet Gynecol. 1990;162:354-57.

13. Tanahatoe SJ, Hompes PG, Lambalk CB. Investigation of the Infertile Couple. Should Diagnostic Laparoscopy be Performed in the Infertility Work up Program in Patients Undergoing Intrauterine Insemination? Human Reproduction. 2003;18:8-11.

14. Rowe PJ, Comhaire FH, Hargreave TB, Mellows HJ. WHO Manual for the Standardized Investigation and Diagnosis of the Infertile Couple. 1993. 